\title{
Conhecendo as mulheres que realizaram o exame Papanicolau em uma Unidade Básica de Saúde de uma cidade de Minas Gerais
}

\author{
Waldere Fabri Pereira Ribeiro, D.Sc.*, Gabrielly Martins da Silva**, Verônica dos Santos Rezende**
}

*Enfermeira, docente da Escola de Enfermagem Wenceslau Braz, ItajubálMG, ${ }^{* *}$ Graduanda do $7^{\circ}$ periodo do curso de Graduação em Enfermagem da Escola de Enfermagem Wenceslau Braz, Itajubá/MG

\begin{abstract}
Resumo
Trata-se de um estudo descritivo, documental e retrospectivo com abordagem quantitativa que teve como objetivo conhecer as características pessoais e ginecológicas das mulheres que realizaram o exame Papanicolau na Unidade Básica de Saúde do Bairro Novo Horizonte, em Itajubá, Minas Gerais. A amostra foi constituída por 58 prontuários das pacientes que colheram material para o referido exame no período compreendido entre maio de 2011 e abril de 2012. A coleta de dados foi realizada pelas pesquisadoras nos meses de junho e julho de 2012, na referida unidade. Resultados encontrados: 55,17\% das mulheres estavam na faixa etária de $16-25$ anos; $50 \%$ eram casadas; $63,79 \%$ eram católicas; $50 \%$ possuíam o ensino fundamental; 44,83\% eram do lar. Quanto às características ginecológicas, 34,48\% apresentaram menarca na faixa etária de 10-12 anos; 25,86\% com coitarca na faixa etária de 15-16 anos; 62,07\% com ciclo menstrual regular; 69,23\% tiveram corrimento como alteração vaginal; $93,10 \%$ não tinham DST; $87,93 \%$ sem alteração nas atividades sexuais; $60,34 \%$ faziam o uso de método contraceptivo e $91,38 \%$ tinham antecedentes obstétricos. Os resultados demonstram que se pode oferecer subsídios para a implantação de espaços de discussão e reflexão sobre essas mulheres, com o objetivo de oferecer a elas atendimento de enfermagem específico direcionado às necessidades básicas identificadas.
\end{abstract}

Palavras-chave: saúde da mulher, Enfermagem, prevençāo primária, câncer uterino.

\section{Abstract \\ Knowing women who performed the Papanicolaou test in a Health Basic Unit of a city in Minas Gerais}

This is a descriptive, documentary and retrospective study with quantitative approach which aimed to understand personal and gynecological characteristics of women who underwent to the Papanicolaou screening test in the Health Basic Unit of Novo Horizonte neighborhood in the city of Itajubá, Minas Gerais. The sample was composed of 58 patient charts who gathered material for the mentioned examination between May 2011 and April 2012. Data collection was performed during June and July 2012 at the unit. Results: $55.17 \%$ of women were aged $16-25$ years, $50 \%$ were married, $63.79 \%$ were catholic, $50 \%$ had elementary school, $44.83 \%$ were housewives. Regarding the gynecological characteristics, $34.48 \%$ had the menarche at $10-12$ years, $25.86 \%$ with first sexual intercourse at the age of $15-16$ years, $62.07 \%$ with regular menstrual cycles, $69.23 \%$ had discharge as vaginal changes; $93.10 \%$ had no STD; $87.93 \%$ without changes in sexual 
activities; $60.34 \%$ were using contraceptive methods and $91.38 \%$ had obstetric antecedents. The conclusion, as the results demonstrated, is that it is possible to provide allowances for discussion and reflection opportunities about these women, in order to offer them specific nursing care directed to the identified basic needs.

Key-words: women's health, nursing, primary prevention, cervix neoplasms.

\section{Resumen}

\section{Conociendo mujeres que realizaron la prueba de Papanicolaou en una Unidad Básica de Salud de una ciudad en Minas Gerais}

Este es un estudio descriptivo, documental y retrospectivo con abordaje cuantitativo que tuvo como objetivo identificar las características personales y ginecológicas de mujeres que se sometieron a frotis de Papanicolaou en la Unidad Básica de Salud del Bairro Novo Horizonte, en Itajubá, Minas Gerais. La muestra estuvo conformada por 58 historias clínicas de pacientes que recogieron material para el mencionado examen entre mayo de 2011 y abril de 2012. La recolección de datos se llevó a cabo por los investigadores durante los meses de junio y julio de 2012, en la referida unidad. Resultados del estudio: El 55,17\% de las mujeres tenían entre 16-25 años, el 50\% estaban casadas, el 63,79\% eran católicas, el $50 \%$ tenían enseńanza básica, el 44,83\% eran amas de casa. En cuanto a las características ginecológicas, 34,48\% tuvo la menarquia entre $10-12$ ańos, $25,86 \%$ con primera relación sexual entre $15-16$ ańos, $62,07 \%$ con ciclos menstruales regulares, el 69,23\% tenía corrimiento como alteraciones vaginales; el 93,10\% no tenía enfermedades de transmisión sexual; el 87,93\% sin cambios en actividades sexuales, el 60,34\% estaba usando anticonceptivos y el 91,38\% tenía historia obstétrica. Los resultados demuestran que se puede ofrecer subsidios para la implantación de espacios de discusión y reflexión sobre estas mujeres, con el fin de ofrecerles atención de enfermería específicos dirigidos a necesidades básicas identificadas.

Palabras-clave: salud de la mujer, Enfermería, prevención primaria, cáncer uterino.

\section{Introdução}

O câncer de colo de útero é considerado, no Brasil, um problema de Saúde Pública, pois atinge todas as camadas sociais e regióes geoeconômicas [1]. É o segundo tipo de câncer mais comum entre as mulheres e responsável por quase 230 mil óbitos por ano [2]. Este tipo de câncer é a segunda neoplasia maligna mais comum, superada apenas pelo câncer de mama [3]. Por apresentar uma evoluçáo lenta, o câncer de colo de útero se torna evitável, já que há um longo período desde o desenvolvimento das lesóes precursoras até o aparecimento do câncer [4].

O câncer uterino possui um fácil rastreamento, pois as suas alteraçôes neoplásicas são diagnosticadas com um teste simples, o exame preventivo de câncer de colo de útero, também conhecido pelo nome de exame Papanicolau [5]. Esse exame tem como objetivo detectar o câncer do colo do útero e suas lesóes precursoras o mais rapidamente possível. Trata-se de uma medida preventiva que é responsável pelo declínio da incidência e da mortalidade do câncer cervical nos últimos 50 anos [5]. O rastreamento de câncer uterino, por meio do exame Papanico- lau, em mulheres na faixa etária de 25 a 65 anos, reduz cerca de $80 \%$ da mortalidade por este tipo de câncer. Além disso, para garantir essa redução, é necessário organização, integralidade e qualidade do rastreamento e acompanhamento das pacientes no tratamento [6].

Acredita-se que atender a mulheres que tenham o interesse em realizar o exame Papanicolau é um papel relevante da enfermagem na promoção da saúde, um modo de atender essas mulheres e uma ótima oportunidade para que recebam esclarecimentos necessários sobre o assunto. A Enfermagem, no momento que atende as necessidades individuais da paciente, esclarecendo e retirando possíveis dúvidas em relação à saúde da mulher, está promovendo melhorias na qualidade de vida das pacientes.

\section{Material e métodos}

Este estudo caracteriza-se como quantitativo, descritivo, documental, retrospectivo, fundamentado na análise de um documento denominado "Consulta de Enfermagem - Ginecológica" que compunha o prontuário das pacientes que colheram material 
para o exame Papanicolau na UBS do Bairro Novo Horizonte no período de maio de 2011 a abril de 2012.

A pesquisa descritiva tem como objetivo primordial a descrição das características de determinada população ou fenômeno ou, então, o estabelecimento de relações entre variáveis [7].

Outra característica deste tipo de pesquisa é que uma vez observados os fatos estes são registrados, analisados, classificados e interpretados, sem que o pesquisador interfira neles. Isto significa que os fenômenos do mundo físico e humano são estruturados, mas não manipulados pelo pesquisador [8].

$\mathrm{Na}$ pesquisa documental, as fontes são mais diversificadas e dispersas como documentos conservados em arquivos de órgãos públicos e instituiçóes de ordem privada, assim como os documentos que foram analisados nesta pesquisa. Entre suas vantagens podemos citar: é uma fonte rica e estável de dados, possui baixo custo e náo existe contato direto com os sujeitos da pesquisa [7]. O projeto foi autorizado e encaminhado ao Comitê de Ética e Pesquisa (CEP) da Escola de Enfermagem Wenceslau Braz, para apreciação, e foi aprovado com o parecer consubstanciado No 529/2011.

\section{Resultados}

Os resultados foram divididos em duas partes e apresentados na Tabela I - Características Pessoais e na Tabela II - Características Ginecológicas.

Tabela I - Características pessoais das pacientes que realizaram o exame Papanicolau, UBS - CAIC, Itajubá, MG, $2011 / 2012$. $(\mathrm{n}=58)$

\begin{tabular}{lcc}
\hline \multicolumn{1}{c}{ Características } & \multicolumn{2}{c}{ Distribuição } \\
\hline Idade em anos & $\mathrm{f}$ & $\%$ \\
\hline $16-25$ & 32 & 55,17 \\
$26-35$ & 11 & 18,97 \\
$36-45$ & 9 & 15,52 \\
$46-55$ & 2 & 3,45 \\
$56-65$ & 4 & 6,90 \\
Acima de 65 & 0 & 0 \\
\hline Estado civil & \multicolumn{3}{c}{} \\
\hline Solteira & 16 & 27,59 \\
Casada & 29 & 50 \\
Viúva & 5 & 8,62 \\
Separada & 7 & 12,07 \\
Amasiada & 1 & 1,72 \\
\hline Religião & & \\
\hline Católica & 37 & 63,79
\end{tabular}

\begin{tabular}{lcc} 
Evangélica & 21 & 36,21 \\
Espírita & 0 & 0 \\
Outras & 0 & 0 \\
\hline Escolaridade & & \\
\hline Analfabeta & 2 & 3,45 \\
Até 4 ${ }^{a}$ série & 4 & 6,90 \\
De 5 a 8 ${ }^{a}$ série & 29 & 50 \\
Ensino Médio Completo & 22 & 37,93 \\
Ensino Superior Completo & 1 & 1,72 \\
Ensino Superior Incom- & & \\
pleto & 0 & 0 \\
\hline Ocupação & & \\
\hline Aposentada & 2 & 3,45 \\
Do lar & 26 & 44,83 \\
Estudante & 11 & 18,97 \\
Outras & 19 & 32,76 \\
\hline & &
\end{tabular}

Tabela II - Características ginecológicas das pacientes que realizaram o exame Papanicolau, UBS - CAIC, Itajubá, MG, 2011 / 2012. (n=58)

\begin{tabular}{|c|c|c|}
\hline \multirow{2}{*}{ Características } & \multicolumn{2}{|c|}{ Distribuição } \\
\hline & $f$ & $\%$ \\
\hline \multicolumn{3}{|l|}{ Menarca } \\
\hline 10-12 anos & 20 & 34,48 \\
\hline 13-14 anos & 17 & 29,31 \\
\hline $15-16$ anos & 14 & 24,14 \\
\hline Acima de 16 anos & 7 & 12,07 \\
\hline \multicolumn{3}{|l|}{ Coitarca } \\
\hline 10-12 anos & 1 & 1,72 \\
\hline 13-14 anos & 8 & 13,79 \\
\hline $15-16$ anos & 15 & 25,86 \\
\hline 17-18 anos & 12 & 20,69 \\
\hline $19-20$ anos & 10 & 17,24 \\
\hline Acima de 20 anos & 12 & 20,69 \\
\hline \multicolumn{3}{|l|}{ Ciclo Menstrual } \\
\hline Irregular & 22 & 37,93 \\
\hline Regular & 36 & 62,07 \\
\hline \multicolumn{3}{|l|}{ Alterações Vaginais } \\
\hline Corrimento & 49 & 84,48 \\
\hline Prurido & 8 & 13,79 \\
\hline Lesão & 1 & 1,72 \\
\hline \multicolumn{3}{|l|}{ DST } \\
\hline Sim & 4 & 6,90 \\
\hline Não & 54 & 93,10 \\
\hline \multicolumn{3}{|l|}{ Qual? } \\
\hline Herpes & 2 & 50 \\
\hline Não sabe & 2 & 50 \\
\hline \multicolumn{3}{|l|}{ Atividade Sexual } \\
\hline Sem alteração & 51 & 87,93 \\
\hline Com alteração & 7 & 12,07 \\
\hline \multicolumn{3}{|l|}{ Qual? } \\
\hline DST & 2 & 3,45 \\
\hline Dor & 3 & 5,17 \\
\hline \multicolumn{3}{|l|}{ Diminuição de lubrifica- } \\
\hline ção vaginal & 2 & 3,45 \\
\hline
\end{tabular}




\begin{tabular}{lcc}
\hline $\begin{array}{l}\text { Uso de Método Contra- } \\
\text { ceptivo }\end{array}$ & & \\
\hline Sim & 35 & 60,34 \\
Não & 23 & 39,66 \\
Qual? & & \\
Anticoncepcional oral & 15 & 42,86 \\
Anticoncepcional injetável & 3 & 8,57 \\
Tabela & 3 & 8,57 \\
Preservativo masculino & 8 & 22,86 \\
Outro & 6 & 17,14 \\
\hline Antecedentes Obstétricos & & \\
\hline Sim & 53 & 91,38 \\
Não & 5 & 8,62 \\
Qual? & & \\
Gestação & 81 & 48,80 \\
Partos & 70 & 42,17 \\
Abortos & 15 & 9,04 \\
\hline
\end{tabular}

\section{Discussão}

$\mathrm{Na}$ análise dos dados pessoais, em relação à idade observamos que houve uma prevalência de $32(55,17 \%)$ mulheres na faixa etária de 16 a 25 anos, seguida de $11(18,97 \%)$ com 26 a 35 anos e nenhuma acima de 65 anos. O exame Papanicolau deve ser realizado em mulheres de 25 a 60 anos de idade [9]. Esse limite de idade foi aumentado para 64 anos no ano de 2011 [10]. A faixa etária na qual tivemos o maior percentual é considerada como uma faixa etária em que a incidência do câncer é alarmante [1]. Com a inicialização da atividade sexual cada vez mais precoce e muitas vezes desprotegida, faz-se necessário a realização do papanicolau por parte desta faixa etária devido a sua suscetibilidade [11]. A frequência foi menor nas faixas etárias acima de 46 anos. Esta baixa procura do exame preventivo por mulheres de maior idade se deve ao fato de que estas já não se encontram mais na faixa etária produtiva, muitas delas se deparando com a menopausa, cabendo então aos serviços de saúde implementar açôes que atinjam essa parte da população [12].

O estado civil predominante foi o de casada com 29 (50\%) e este fato é esclarecido na literatura que fala que as mulheres sem companheiros buscam os serviços de saúde com menor frequência em relação as que possuem parceiro fixo, tornando-as então mais passíveis de desatualização na realização do preventivo [13].

No que concerne a religião, $37(63,79 \%)$ mulheres seguiam a religiáo católica e 21 (36,21\%) a evangélica. A influência da religiáo quanto a esta questão está no fato de que muitas instituições proporcionam às mulheres um incentivo a hábitos saudáveis, apoio psicológico, reforçando a autoestima e estruturando-as para suportarem eventos da vida [14].

Quanto à escolaridade, 29 (50\%) mulheres tinham somente o ensino fundamental, seguida de $22(37,93 \%)$ com ensino médio completo. O nível de escolaridade é fator importante para medidas preventivas quanto ao câncer de colo de útero, visto que o analfabetismo e o baixo nível educacional podem impedir ou dificultar a informação sobre a doença, sobre a utilização dos serviços de saúde, sobre os fatores de risco, sobre a importância do exame preventivo e do esclarecimento de possíveis dúvidas [15]. Quanto mais elevado for o nível de escolaridade maior será a importância dada por parte destas mulheres a realização do Papanicolau, bem como a compreensão de sua realização conforme preconizado pelo Ministério da Saúde [16].

Em relação à ocupação, $26(44,83 \%)$ das mulheres eram do lar, seguida de 19 (32,76\%) que tinham outras ocupaçóes. Este item está juntamente relacionado com a questáo da escolaridade, uma vez que a maioria destas mulheres tem como ocupação atual a do lar que é uma atividade que geralmente requer uma baixa escolaridade para ser realizada [12].

Analisando as características ginecológicas, observamos que a menarca em 20 mulheres (34,48\%) ocorreu entre 10-12 anos, seguida de 17 mulheres (29,31\%) que ocorreu entre 13-14 anos e que a menarca precoce, aquela que se dá antes dos 12 anos, é tida como um dos fatores de risco para o desenvolvimento do câncer do colo uterino [17]. Faz-se entáo necessário uma maior atenção quanto à ocorrência do câncer do colo do útero cada vez mais cedo devido à antecipação da iniciação da puberdade. Esse decréscimo na idade da primeira menstruação consequentemente faz com que aconteça a inicializaçáo precoce da atividade sexual e surge a capacidade reprodutiva. Por isso, é importantíssimo conscientizar estas jovens quanto à realização de estratégias de prevenção ao câncer de colo de útero, como o exame Papanicolau [9]. Porém para alguns autores a menarca não apresentou significância estatística quando relacionado à realização do exame [18].

Em relação à coitarca observamos que em 15 mulheres $(25,86 \%)$ a mesma ocorreu entre os $15-16$ anos, seguida de $12(20,69 \%)$ mulheres que tiveram sua primeira relação sexual 17-18 anos sendo a precocidade sexual um dos fatores de risco para o 
desenvolvimento do câncer de colo do útero devido à imaturidade do epitélio e a sua suscetibilidade as agressóes oncogênicas [19].

Em análise ao ciclo menstrual 36 mulheres $(62,07 \%)$ tinham o seu ciclo regular, enquanto que 22 mulheres $(37,93 \%)$ tinham o ciclo irregular. A questão da irregularidade do ciclo menstrual é um dos principais motivos que leva as mulheres a realizarem o exame Papanicolau, talvez pela preocupação ou porque conhece alguém que apresentou este episódio e posteriormente descobriu alguma alteração no exame [20].

Das mulheres pesquisadas, 35 (60,34\%) afirmam que utilizaram algum tipo de método contraceptivo e $23(39,66 \%)$ delas informaram não utilizar. Dentre os métodos utilizados, 15 (42,86\%) escolheram o anticoncepcional oral; 8 (22,86\%) o preservativo masculino; $3(8,57 \%)$ o anticoncepcional injetável e o método da tabela e $6(17,14 \%)$ outros métodos. O contraceptivo oral geralmente é utilizado por mulheres sexualmente ativas levando-as a não utilizar outro método de barreira, estando assim mais expostas a contrair o HPV. Por outro lado, observa-se também que essas mulheres recorrem à consulta ginecológica, possibilitando, desta forma, um rastreamento mais eficaz para o câncer do colo do útero [20]. As mulheres que fazem uso de métodos contraceptivos, por acabarem frequentando mais vezes as unidades de saúde, buscando este planejamento familiar, acabam sendo orientadas a fazer o exame Papanicolau conforme preconizado pelo Ministério da Saúde [21].

Sobre as Doenças Sexualmente Transmissíveis (DST) 54 (93,10\%) mulheres afirmaram não ter e 4 $(6,90 \%)$ delas disseram já ter tido. Dessas mulheres $2(50 \%)$ disseram ter tido Herpes e outras 2 (50\%) náo saber qual era a doença. Quando presente uma DST, esta pode provocar o aparecimento de lesóes ou inflamaçóes na pele ou nas mucosas genitais que, caso não sejam tratadas, poderão se tornar lesóes pré-cancerosas que podem levar a um câncer de colo uterino futuramente [20].

Em relação às alteraçóes vaginais, verificamos que $18(69,23 \%)$ mulheres apresentavam corrimento, $7(26,92 \%)$ prurido e $1(3,85 \%)$ lesão. O corrimento é um dos problemas mais comuns que afeta as mulheres, conhecido também como vaginite, responsável pela procura mais frequente da mulher à unidade de saúde. Esse corrimento pode ou não ter um odor desagradável, pode ocorrer também ardor, coceira ou até mesmo vontades mais frequentes de urinar. Essas alteraçôes podem ser decorrentes de infecçóes vaginais ou cervicais do colo do útero ou por DST [20].

Analisando as alteraçóes durante as atividades sexuais dessas mulheres, 51 (87,93\%) delas disseram náo ter qualquer alteraçáo, enquanto que $3(5,17 \%)$ relataram dor, 2 (3,45\%) alteração devido a DST e 2 $(3,45 \%)$ tiveram alteração que consideram ter sido decorrente da diminuição da lubrificação vaginal. Os sinais relatados acima devem ser levados em consideração, pois a maioria das que apresentam algumas dessas alteraçóes não procuram tratamento, ou por julgarem ser uma condição normal ou por serem situaçôes pouco sérias, e em consequência quanto mais se demora a procurar o tratamento adequado, maiores e mais sérias são as complicaçóes [20].

Quanto aos antecedentes obstétricos, $53 \mathrm{mu}-$ lheres $(91,38 \%)$ disseram ter antecedente obstétrico, seguido de 5 mulheres $(8,62 \%)$ que não possuíam antecedente obstétrico. Atualmente pode-se confirmar o papel da alta paridade ao câncer cervical em que há relaçóes com o aumento significativo dos números de câncer cervical ou neoplasias epiteliais de alto grau com o número elevado de filhos, e que este fato se daria devido ao estado nutricional, hormonal, traumatismo e mecanismos imunológicos que sofrem alguma alteração no decorrer da gestação [22].

\section{Conclusão}

Concluímos que em relação às características pessoais, 55,17\% estavam na faixa etária de 16-25 anos; $50 \%$ eram casadas; $63,79 \%$ eram católicas; $50 \%$ possuíam o ensino fundamental e $44,83 \%$ eram do lar. Já em relação às características ginecológicas, 34,48\% com menarca na faixa etária de 10-12 anos; $25,86 \%$ com coitarca na faixa etária de 15-16 anos; $62,07 \%$ com ciclo menstrual regular; 69,23\% tiveram corrimento como alteração vaginal; 93,10\% não tinham DST; $87,93 \%$ sem alteração nas atividades sexuais; $60,34 \%$ faziam o uso de método contraceptivo e $91,38 \%$ tinham antecedentes obstétricos. Ao atingir o objetivo proposto no início da pesquisa e conhecer o perfil das mulheres que realizaram a coleta do material citológico para o exame preventivo do câncer de colo de útero na Unidade Básica de Saúde do Bairro Novo Horizonte, pode-se afirmar que essas mulheres usufruíram de um direito à saúde enquanto cidadãs, desenvolvendo desta forma um cuidado de saúde para consigo mesmas. 


\section{Referências}

1. Bim CR, Pelloso SM, Carvalho MDB, Previdelli ITS. Diagnóstico precoce do câncer de mama e colo uterino em mulheres do município de Guarapuava. Rev Esc Enferm 2010:44(4):940-46.

2. Ministério da Saúde (BR). Manual de atenção a mulher no climatério/menopausa. Brasília: MS; 2008.

3. Albuquerque KM, Paulo Germano Frias PG, Andrade CLT, Aquino EML, Menezes G, Szwarcwald CL. Cobertura do teste de Papanicolau e fatores associados à não-realizaçáo: um olhar sobre o Programa de Prevenção do Câncer do Colo do Útero em Pernambuco, Brasil. Cad Saúde Pública 2009;25(2):301-9.

4. Gonzaga CMR, Freitas-Junior R, Barbaresco AA, Martins E, Bernardes BTIV, Resende APM. Cervical cancer mortality trends in Brazil: 1980-2009 Cad Saúde Pública 2013;29(3):599-608.

5. Vasconcelos CTM, Castelo ARP, Medeiros FC, Pinheiro AKB. Análise da cobertura e dos exames colpocitológicos não retirados de uma Unidade Básica de Saúde. Rev Esc Enferm USP 2010;44(2):324-30.

6. Deus CA. O papel do enfermeiro na prevenção do câncer do colo do útero em unidade básica de saúde com equipe de saúde da família [Monografia]. Uberaba: UFMG; 2011.

7. Bifulco VA, Fernandes Júnior HJ, Barboz AB. Câncer: uma visão multiprofissional. Barueri: Manole; 2010.

8. Gil AC. Como elaborar projetos de pesquisa. $5^{a}$ ed. São Paulo: Atlas; 2010.

9. Oliveira PS. Metodologia das ciências humanas. São Paulo: Hucitec/UNESP; 1998.

10. Instituto Nacional do Câncer. Ações de enfermagem para o controle do câncer: uma proposta de integração ensino-serviço. 3a. ed. Rio de Janeiro: INCA; 2008.

11. Instituto Nacional do Câncer. Programa Nacional de Controle do Câncer do Colo do Útero. Rio de Janeiro: INCA; 2012.

12. Ferreira MLSM. Motivos que influenciam a não-realização do exame de papanicolaou segundo a percepção de mulheres. Esc Anna Nery Rev Enferm 2009;13(2):378-84.
13. Melo MCSC, Vilela F, Salimena AMO, Souza IEO. O enfermeiro na prevençáo do câncer do colo do útero: o cotidiano da atenção primária. Rev Brasil Cancerol 2012;58(3):389-98.

14. Wiesner-Ceballos C, Vejarano-Velandia M, Caicedo-Mera JC, Tovar-Murillo S, Cendales-Duarte. La citología de cuello uterino en Soacha, Colombia: representaciones sociales, barreras y motivaciones. Rev Salud Publica (Bogota);8(3):185-196.

15. Lucena LT, Zãn DG, Crispim PTB, Ferrari JO. Fatores que influenciam a realização do exame preventivo do câncer cérvico-uterino em Porto Velho, Estado de Rondônia, Brasil. RevPan-Amaz 2011;2(2):45-50.

16. Jorge RJB, Diógenes MAR, Mendonça FAC, Sampaio LRL. Exame Papanicolaou: sentimentos relatados por profissionais de enfermagem ao se submeterem a esse exame. Ciênc Saúde Coletiva 2011;16(5):2443-51.

17. Soares MB, Silva SR. Analysis of a municipal program of uterine cervical neoplasm prevention Rev Bras Enferm 2010;63(2):177-82.

18. Nascimento MI, Monteiro GTR. Características de acesso ao preventivo de câncer de colo do útero: três etapas metodológicas da adaptação do instrumento de coleta de informação. Cad Saúde Pública 2010;26(6):1096-108.

19. Muller DK, Dias-da-Costa JS, Luz AMH, Olinto MTA. Cobertura do exame citopatológico do colo do útero na cidade de São Leopoldo, Rio Grande do Sul, Brasil. Cad Saúde Pública 2008;24(11):2511-20.

20. Reis AAS, Monteiro CD, Paula CB, Santos RS, Saddi VA, Cruz AD. Papilomavírus humano e saúde pública: prevenção ao carcinoma de cérvice uterina. Ciênc Saúde Coletiva 2010;15(1):1055-60.

21. Casarin MR, Piccoli JCE. Educação em saúde para prevençáo do câncer de colo do útero em mulheres do município de Santo Ângelo/RS. Ciênc Saúde Coletiva 2011;16(9):3925-32.

22. Fernandes JV, Rodrigues SHL, Costa YGAS, Silva LCM, Brito AML, Azevedo JWV, et al. Conhecimentos, atitudes e prática do exame de Papanicolaou por mulheres, Nordeste do Brasil. Rev Saúde Pública 2009;43(5):851-8. 